\section{Molecular Syndromology}

Mol Syndromol 2018;9:110-114

DOI: $10.1159 / 000485908$
Accepted: November 11, 2017

by M. Schmid

Published online: January 18, 2018

\title{
A Novel GMPPA Mutation in Two Adult Sisters with Achalasia, Alacrima, Short Stature, Dysmorphism, and Intellectual Disability
}

\author{
Edmar O. Benítez ${ }^{a}$ Juan J. Morales ${ }^{a}$ Luis A. Muñoz ${ }^{a}$ Christian A. Hübner ${ }^{b}$ \\ Osvaldo M. Mutchinick ${ }^{a}$ \\ a Department of Genetics, Instituto Nacional de Ciencias Médicas y Nutrición "Salvador Zubirán", Mexico City, \\ Mexico; ${ }^{b}$ Institute of Human Genetics, Jena University Hospital, Jena, Germany
}

\section{Keywords}

AAMR syndrome $\cdot$ GMPPA $\cdot$ Novel mutation

\begin{abstract}
The alacrima, achalasia, and mental retardation syndrome (AAMR) is a newly described autosomal recessive disorder characterized by the onset of these 3 main features at birth or in early infancy. At present, only 16 cases have been reported. Recently, it was shown that AAMR is due to mutations in the guanosine diphosphate (GDP)-mannose pyrophosphorylase A (GMPPA) gene. These mutations induce a significant GDP-mannose overload, which may affect protein glycosylation. Herein, for the first time, we describe 2 adult sisters with AAMR with a previously not reported deleterious homozygous missense mutation c.1118G $>C$ (p.Arg373Pro) in the GMPPA gene, born to healthy consanguineous heterozygous parents from an ancient endogamous population. The main symptoms in both sisters started soon after birth with achalasia and feeding difficulties, requiring surgical treatment. Both sisters showed alacrima identified during the first months of life, delayed psychomotor development, speech delay, facial dysmorphism, limb
\end{abstract}

() 2018 S. Karger AG, Basel defects, short stature, and moderate intellectual disability. Alacrima and feeding difficulties due to achalasia during the neonatal period or first months of life, in the absence of adrenal cortical insufficiency, should spur to investigate AAMR by sequencing the GMPPA gene.

(c) 2018 S. Karger AG, Basel

Alacrima, achalasia, and mental retardation syndrome (AAMR) (OMIM 615510; http://www.omim.org) is a very rare autosomal recessive disease characterized by this symptomatic triad at birth or during the first months of life. Less frequent phenotypic characteristics are hypotonia, gait abnormalities as well as visual and hearing deficits. Clinically, it overlaps with triple-A syndrome (OMIM 231550) which is characterized by the same triad, but distinct to AAMR, since it also presents with adrenocortical insufficiency [Allgrove et al., 1978]. Koehler et al. [2013] were the first to report homozygous mutations in the GMPPA gene, located at chromosome band $2 \mathrm{q} 35$, in 13 patients from 9 unrelated families presenting with alacrima, feeding difficulties due to achalasia, at birth or in early infancy, and delayed psychomotor development

Prof. Osvaldo M. Mutchinick, MD, PhD

Department of Genetics

Instituto Nacional de Ciencias Médicas y Nutrición "Salvador Zubirán”

Vasco de Quiroga 15, Col. Belisario Domínguez, Mexico City 14080 (Mexico)

E-Mail osvaldo@unam.mx 
including speech disorders. None of these patients had mutations in the $A A A S$ gene or signs of Addison disease [Dumic et al., 2012]. Immunoblot analysis of cells from patients with reported missense mutations showed lower levels of GMPPA compared to controls, consistent with loss of gene function [Koehler et al., 2013; Gold et al., 2017]. GMPPA is a largely uncharacterized homolog of GMPPB, which catalyzes the formation of guanosine diphosphate (GDP)-mannose, an essential precursor of glycan moieties of glycoproteins and glycolipids. Surprisingly, GDP-mannose pyrophosphorylase activity was normal, and GDP-mannose levels were significantly higher, whereas other nucleotide diphosphate sugars were unchanged. It is assumed that deviations induced by increased GDP-mannose levels have a significant effect in restricted cell types, such as autonomic nerve fibers innervating the distal esophageal sphincter, lacrimal glands, and may affect protein glycosylation leading to disturbances in the levels of guanine nucleotides [Koehler et al., 2013].

Herein, we report the clinical and molecular features of 2 adult Mexican sisters diagnosed in infancy with achalasia as well as alacrima, and later with intellectual disability, short stature, and facial dysmorphism. Both are homozygous for a novel deleterious missense mutation in the GMPPA gene.

We also compare the clinical and molecular findings of this very rare disease with, to our knowledge, only 16 previously reported cases [Koehler et al., 2013; Alabdullatif et al., 2017; Gold et al., 2017].

\section{Clinical Reports}

\section{Patient 1 (V-7)}

The proposita is the younger sibling of a sibship of 5 females and 1 male born alive to consanguineous third-cousin healthy parents, with an additional pregnancy lost at 28 weeks of gestation. The mother was 44 and the father 49 years old at the time of conception of the index patient. She was the outcome of a pregnancy complicated by a threatened abortion at 20 weeks gestation, resolved with bed rest. Delivery was by cesarean section at term. At birth, she presented with hypotonia and respiratory distress. The birth weight was $3,700 \mathrm{~g}$. The length and OFC are unknown. At birth, she had multiple café-au-lait spots as well as axillary and inguinal freckling. She was diagnosed with neurofibromatosis type 1 (NF1). Soon after, she developed feeding difficulties due to esophageal stenosis and achalasia, necessitating surgical treatment at 2 months of age. Alacrima was evident at that time and delayed psychomotor development became apparent during the following months. Other salient phenotypic characteristics during childhood were bilateral horizontal nystagmus, speech delay, nasal voice, moderate hearing impairment, and slight intellectual disability.
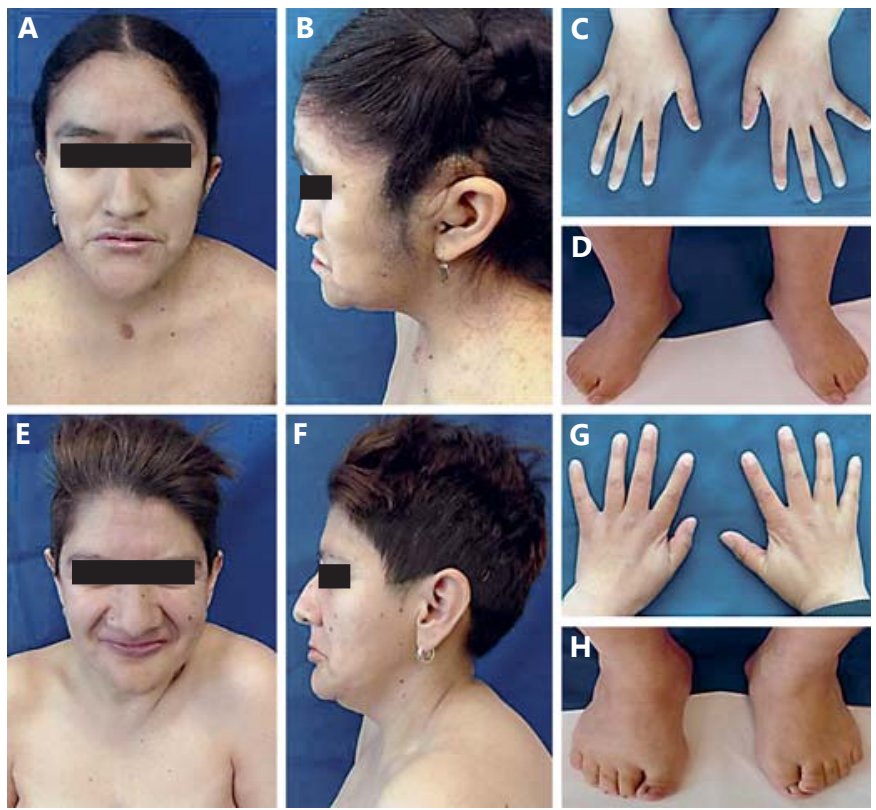

Fig. 1. A-D Sister 1 (V-7). Multiple café-au-lait spots and plexiform neurofibroma at sternal level (A), the left temporoparietal area, and left ear (B). Fusiform fingers and apparent bilateral hypoplasia of thumbs in both sisters $(\mathbf{C}, \mathbf{G})$ and wide feet with bilateral brachydactyly of toes (D, H). E, F Facial images of sister 2 (V-1). Mild facial dysmorphism of both sisters (A, B, E, F).

When first referred to the Genetics Clinic at 28 years of age, she showed generalized muscular hypotonia. Her height was $141 \mathrm{~cm}$ ( $<3 \mathrm{rd}$ centile), weight $49.5 \mathrm{~kg}$ (10-25th percentile), BMI 24.9, and a small head with an OFC of $53 \mathrm{~cm}$ (10th centile). She has an asymmetric triangular face, protruding chin, prominent forehead, high anterior hairline, right eye divergent strabismus, a prominent nose, a short philtrum, adontia, downturned corners of the mouth as well as a prominent helix and antihelix. She also presented with multiple café-au-lait spots $0.5-2 \mathrm{~cm}$ diameter distributed over her thorax and abdomen and a large one covering the lower half of the left thigh. She also has axillary and inguinal freckling as well as plexiform neurofibromas at the sternal angle, the left temporal region, and left ear. She has bilateral clinodactyly of the fifth finger and very short toes with nail hypoplasia (Fig. 1A-D). Hands and thorax X-ray images showed low bone mineral density for her age.

\section{Patient $2(V-1)$}

The girl was born at term by vaginal delivery. Birth weight, length, and OFC are unknown. Similar to her sister, she had perinatal generalized muscular hypotonia and respiratory distress as well as feeding difficulties due to esophageal stenosis and achalasia. She also received surgical treatment at 2 months of age. Alacrima, bilateral horizontal nystagmus, delayed psychomotor development, and speech delay were present some months later.

She was 47 years old when first examined at the Genetics Clinic showing generalized muscular hypotonia. Her height was 138 $\mathrm{cm}$ (<3rd centile), weight $47.3 \mathrm{~kg}$ (25th percentile), BMI 24.8 , and an OFC of $54 \mathrm{~cm}$ (25th centile). She has similar characteristics as 
Fig. 2. Family pedigree and electropherograms of the proposita (V-7), her sister (V1), her father, and mother. Parents are third cousins and carriers of the novel GMPPA mutation c.1118G $>\mathrm{C}$ in exon 11.

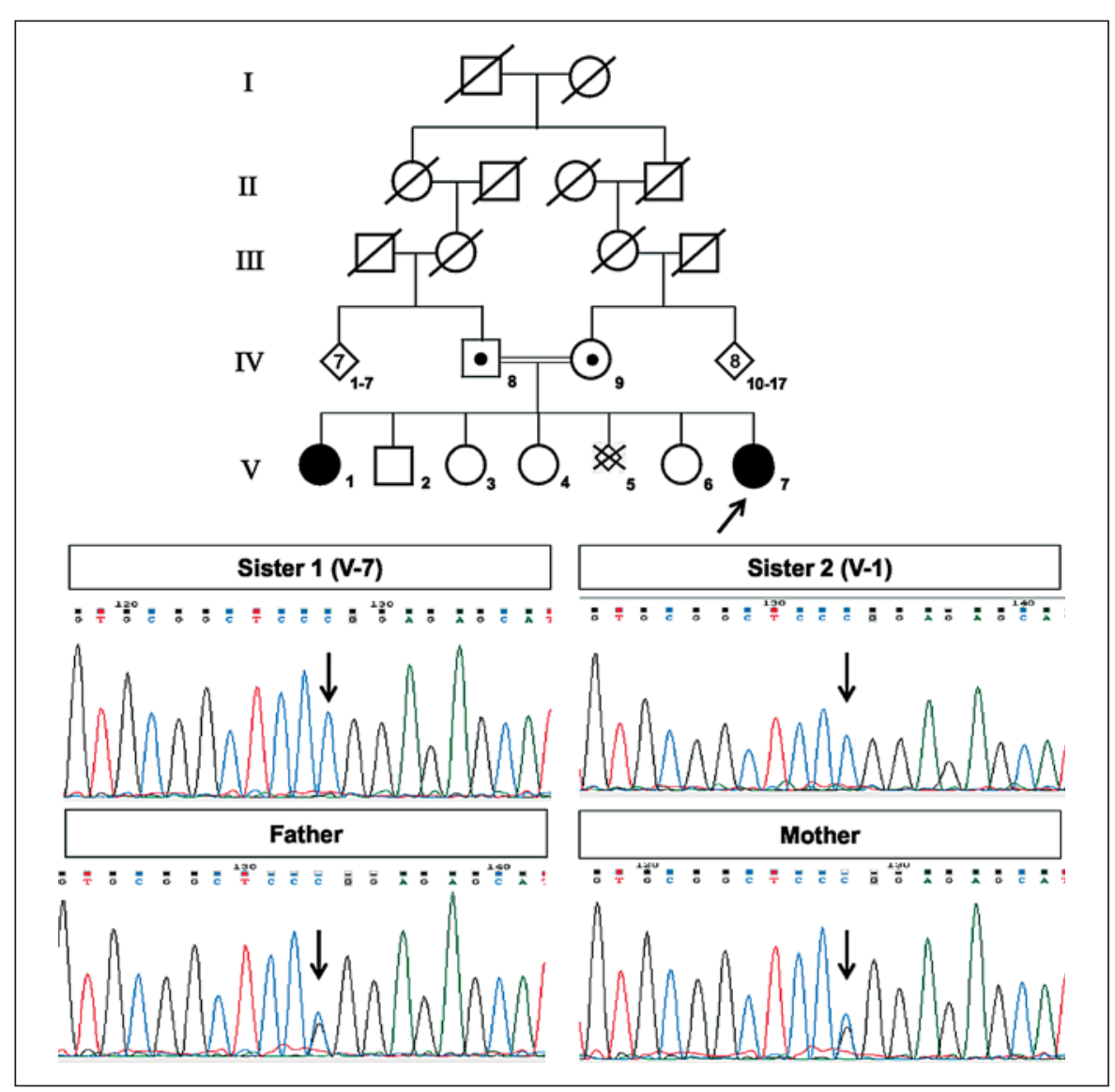

her sister such as the triangular asymmetric face, protruding chin, high anterior hairline, right eye divergent strabismus, prominent nose, short philtrum, downturned corners of the mouth, and adontia. She also has bilateral clinodactyly of the 5th fingers and very short toes with nail hypoplasia. Chest X-ray showed slight cardiomegaly, left-side dorsal scoliosis and osteopenia. Unlike her sister, she did not present with phenotypic features of NF1 (Fig. 1E-H).

\section{Methods and Results}

After parents signed consent, genomic DNA samples of both sisters and their parents were obtained from peripheral blood leukocytes by standard procedures. All exons and flanking sequences of the GMPPA and NF1 genes were sequenced. Both sisters were found to be homozygous for a novel missense mutation in exon 11 of the GMPPA gene. The mutation (c.1118G $>C$ ) results in a p.Arg373Pro amino acid substitution that has not been reported previously and confirmed the clinical diagnosis of AAMR for both sisters. Both parents are heterozygous carriers of the same novel mutation. The family's pedigree and sequencing results of the GMPPA gene are shown in Figure 2. The MutationTaster (http:// www.mutationtaster.org/), SIFT blink (http://sift.jcvi.org), and PolyPhen-2 (http://genetics.bwh.harvard.edu/pph2) predict that the mentioned missense mutation is deleterious and disease caus- ing. The identified novel mutation is not present in the $1000 \mathrm{Ge}$ nomes Project database (http://www.1000genomes.org/).

STRUM (http://zhanglab.ccmb.med.umich.edu/STRUM/) was used to investigate a possible protein stability change induced by the p.Arg373Pro amino acid substitution, by measuring Gibbs's free-energy differences ( $\mathrm{ddG}$ ) between the wild-type and the mutated protein. This analysis showed a ddG of -1.48 which seems to reduce the protein stability. Based on this finding, we propose that this novel mutation has a deleterious loss-of-function effect on the protein.

The proband's (V-7) DNA was also analyzed on a cancer panel that revealed a known deleterious mutation in the NF1 gene, c.6579+1G>A (NM 000267) in exon 34, confirming the diagnosis of NF1 in this patient [Lee et al., 2006]. The mutation was absent in her parents and sister and was therefore interpreted as a de novo event.

\section{Discussion}

This report describes the clinical and molecular features of the first 2 adult patients, 28 and 47 years of age, with AAMR, a very rare autosomal recessive disease of which only 16 cases in early infancy have been previously 
Table 1. Summary of clinical symptoms from 16 cases of AAMR due to GMPPA gene mutations, previously reported and present patients

\begin{tabular}{lllll}
\hline Main symptoms & $\begin{array}{l}\text { Reported } \\
\text { cases }\end{array}$ & $\begin{array}{l}\text { Patient-1 } \\
(\mathrm{V}-1)\end{array}$ & $\begin{array}{l}\text { Patient-2 } \\
(\mathrm{V}-7)\end{array}$ & Total \\
\hline GMPPA mutation & $16 / 16$ & + & + & $18 / 18$ \\
Alacrima & $15 / 15$ & + & + & $17 / 17$ \\
Achalasia & $13 / 15$ & + & + & $15 / 17$ \\
Intellectual disability & $15 / 15$ & + & + & $17 / 17$ \\
Milestones delayed & $15 / 15$ & + & + & $17 / 17$ \\
Speech delayed & $11 / 15$ & + & + & $13 / 17$ \\
Ocular symptoms (myopia, strabismus, & & & $11 / 17$ \\
$\quad$ nystagmus, anisocoria, and not specified) & $9 / 15$ & + & - & $7 / 17$ \\
Gait abnormalities (ataxia, spasticity) & $7 / 15$ & - & + & $6 / 17$ \\
Muscular hypotonia & $4 / 15$ & + & + & $4 / 17$ \\
Hyperkeratosis & $3 / 15$ & - & + & $5 / 17$ \\
Nasal speech & $3 / 15$ & + & + & $5 / 15$ \\
Postural hypotension & $3 / 13$ & + & + & $5 / 15$ \\
Hearing impairment & $3 / 13$ & + & - & $2 / 15$ \\
Hyperreflexia & $2 / 13$ & - & - & $4 / 17$ \\
Sweating decreased & $4 / 15$ & - & - & $2 / 15$ \\
Sensory impairment & $2 / 13$ & - & - & $1 / 15$ \\
Ptosis & $1 / 13$ & - & + & $2 / 15$ \\
Anodontia & NR & + & + & $4 / 17$ \\
Facial dysmorphism & $2 / 15$ & + & + & $5 / 17$ \\
Short stature & $3 / 15$ & + & + \\
\hline
\end{tabular}

AAMR, alacrima, achalasia, and mental retardation syndrome; NR, not previously reported; +, present; -, absent.

described [Koehler et al., 2013; Alabdullatif et al., 2017; Gold et al., 2017]. The sisters reported here are homozygous for a novel deleterious missense mutation in exon 11 in GMPPA (c.1118G $>C$ ) which causes an arginine to proline substitution (p.Arg320Pro) with a predicted pathogenic effect.

Similar to patients reported previously [Koehler et al., 2013; Alabdullatif et al., 2017; Gold et al., 2017], the proposita and her sister also showed neurological symptoms frequently described in AAMR such as delayed milestones, speech delay, and intellectual disability. Additional symptoms were muscular hypotonia, moderate hearing impairment, and nasal speech, characteristics infrequently described in AAMR patients. In contrast to what was reported in some of the previous cases [Koehler et al., 2013], our patients did not have other neurological symptoms such as ataxia, sensory impairment, ptosis, hyperreflexia, spasticity, or gait disturbances.

Interestingly, both sisters had short stature, both below the third percentile for age and growth curves of the general Mexican population, and an expected tallness of $151 \mathrm{~cm}$ according to their midparental height. Two previous reports also described this feature in AAMR patients
[Alabdullatif et al., 2017; Gold et al., 2017]. Gold et al. [2017] observed low bone mineral density for age in a pair of siblings, also present in both of our affected sisters. Potentially these traits are part of the spectrum of the adult AAMR phenotype. Koehler et al. [2013] also described autonomic manifestations in a pair of siblings and another unrelated patient. Our patients only have a mild orthostatic hypotension.

Facial dysmorphism, consisting of an asymmetric face, long pointed chin, strabismus, downturned corners of the mouth, and a prominent helix and antihelix was observed in both sisters and have been recently reported in other patients [Gold et al., 2017]. In reviewing the pictures of 3 brothers from a previous report (Table 1), some facial dysmorphisms observed in our patients were also present in these brothers [Koehler et al., 2013]. Thus, the dysmorphic facial features should be considered as an additional AAMR trait.

Unlike previously published cases [Koehler et al., 2013; Alabdullatif et al., 2017; Gold et al., 2017] that reported patients mostly during infancy, both cases described herein illustrate the phenotype in adult patients. Interestingly, neurological symptoms, intellectual disability, and 
muscular hypotonia remained stable, and neither the proposita nor her sister manifested symptoms of corticoadrenal insufficiency to date.

Regarding the associated neurofibromatosis disease and the confirmed de novo mutation found in the NF1 gene in the proposita, the mutation is probably of paternal origin, considering his advanced age at the time of conception of 49 years of age [Snajderova et al., 2012].

Achalasia, alacrima, and intellectual disability are some of the main characteristics of AAMR; however, similar manifestations plus adrenocortical insufficiency are also the main characteristics of triple-A syndrome. It is possible that AAMR has been misdiagnosed and consequently underreported as some patients previously described as incomplete phenotypes of triple-A syndrome or Allgrove syndrome [Allgrove et al., 1978] may have been AAMR cases due to mutations in the GMPPA gene.

A careful search of the literature identified 2 unrelated cases with features consistent with AAMR, suggesting additional patients exist. The first case was a 12-year-old boy diagnosed with Allgrove syndrome [Allgrove et al., 1978] due to achalasia, alacrima, and neurological dysfunction. However, adrenal function was normal, and neurological examination revealed autonomic dysfunction and intellectual impairment [García-Compeán et al., 1998]. The second case was a 7-year-old girl with alacrima, achalasia, and mental retardation with normal adrenocortical function [Ornek et al., 2002]. Unfortunately, no DNA sequencing was performed on either patient.

During childhood, achalasia, alacrima, psychomotor development delay, and intellectual disability, without adrenocortical insufficiency, may be signs of AAMR, and the GMPPA gene should be sequenced to identify possible mutations. Detailed phenotypic reports of new cases with AAMR, as well as new pathogenic mutations, will help to better define the spectrum and course of the disease and may allow further recognition of genotype-phenotype correlations for many of the features of this syndrome.

Although not yet confirmed, according to the information that this family provided, it is possible that some other affected cases were born in the community. The very small number of families established in the original little village several generations before and the existence of a small number of common private surnames suggests that it was and may still be a highly inbred population, most probably supporting a founder effect for the mutation. At present, we are trying to gain access to the community to identify heterozygous carriers of the mutation and other affected cases to offer genetic counseling to the families living in the village and to other people living nearby.

\section{Statement of Ethics}

Informed consent for manuscript publication and photographs were obtained from the patient's parents, and performed procedures were with the ethical standards.

\section{Disclosure Statement}

The authors have no financial or personal commercial relationships representing a conflict of interest.

\section{References}

Alabdullatif MA, Al Dhaibani MA, Khassawneh MY, El-Hattab AW: Chromosomal microarray in a highly consanguineous population: diagnostic yield, utility of regions of homozygosity, and novel mutations. Clin Genet 91: 616-622 (2017)

Allgrove J, Clayden GS, Grant DB, Macaulay JC: Familial glucocorticoid deficiency with achalasia of the cardia and deficient tear production. Lancet 1:1284-1286 (1978).

Dumic M, Barišic N, Kusec V, Stingl K, Skegro M, et al: Long-term clinical follow-up and molecular genetic findings in eight patients with triple A syndrome. Eur J Pediatr 171:14531459 (2012).
García-Compeán D, Martínez RH, VillegasGonzález MJ, Montes J, García-Quintanilla F, González JA: Achalasia, alacrima without adrenal insufficiency with peripheral and autonomic neurological dysfunction (Allgrove's syndrome) (in Spanish). Rev Gastroenterol Mex 63:33-36 (1998).

Gold WA, Sobreira N, Wiame E, Marbaix A, Van Schaftingen E, et al: A novel mutation in GMPPA in siblings with apparent intellectual disability, epilepsy, dysmorphism, and autonomic dysfunction. Am J Med Genet A 173: 2246-2250 (2017)
Koehler K, Malik M, Mahmood S, Giesselmann S, Beetz C, et al: Mutations in GMPPA cause a glycosylation disorder characterized by intellectual disability and autonomic dysfunction. Am J Hum Genet 93:727-734 (2013).

Lee MJ, Su YN, You HL, Chiou SC, Lin LC, et al: Identification of forty-five novel and twentythree known NF1 mutations in Chinese patients with neurofibromatosis type 1 . Hum Mutat 27:832 (2006).

Ornek K, Atilla H, Zilelioğlu G: Pediatric alacrima, achalasia and mental retardation. J AAPOS 6:261-263 (2002).

Snajderova M, Riccardi VM, Petrak B, Zemova D, Zapletalova J, et al: The importance of advanced parental age in the origin of neurofibromatosis type 1 . Am J Med Genet A 158A:519-523 (2012).

Benítez/Morales/Muñoz/Hübner/ Mutchinick 